\title{
Cosmology and Nonlinear Supersymmetric General Relativity
}

\author{
Kazunari Shima*广 \\ Saitama Institiute of Technology, Japan \\ E-mail: shima@sit.ac.jp
}

We discuss the cosmological implications of nonlinear supersymmetric(NLSUSY) general relativity(GR) of vacuum Einstein-Hilbert(EH) action-type. NLSUSY GR is obtained by the geomtrical arguments on new spacetime just inspired by NLSUSY. New spacetime is unstable and induces spontaneously the phase transition to Riemann spacetime with NG fermion matter. i.e. the new action of NLSUSY GR is unstable and breaks down spontaneously to EH action with Nambu-Goldstone(NG) fermion matter. We show that NLSUSY GR may describe naturally the paradigm of the present expanding universe and elucidate the physical meanings and relations among the cosmologicaly important quantities,e.g., the spontaneous SUSY breaking scale, the cosmological constant, the dark energy and the neutrino mass.

International Europhysics Conference on High Energy Physics

July 21st - 27th 2005

Lisboa, Portugal

* Speaker.

${ }^{\dagger}$ A footnote may follow. 


\section{Nonlinear Supersymmetric General Relativity(NLSUSY GR)}

SUSY and its spontaneous breakdown may be conformed to the rationale of spacetime. The NLSUSY invariant coupling of the graviton with the spin $\frac{1}{2}$ fermion is anticipated for materializing the standard model(SM) with the spontaneous breakdown of SUSY[1]. We extend the geometrical arguments of Einstein general relativity(EGR) on Riemann spacetime to new (called SGM hereafter) spacetime where the tangent spacetime posesses NLSUSY degrees of freedom(d.o.f), i.e. besides the ordinary SO $(3,1)$ Minkowski coordinate $x^{a}$ the SL(2C) Grassman coordinates $\psi$ for the coset space $\frac{\operatorname{super} G L(4, R)}{G L(4, R)}$ turning subsequently to the NG fermion (called superon hereafter) dynamical d.o.f. are attached at every curved spacetime point. (Note that locally homomorphic $\operatorname{SO}(3,1)$ and $\mathrm{SL}(2 \mathrm{C})$ are non-compact groups for spacetime d.o.f analogous to $\mathrm{SO}(3)$ and $\mathrm{SU}(2)$ compact groups for U(1) gauge d.o.f. of 't Hooft-Polyakov monopole.) We have obtained the following NLSUSY GR(N=1 SGM) action[2] of the vacuum EH-type in SGM spacetime.

$$
\begin{gathered}
L(w)=-\frac{c^{4}}{16 \pi G}|w|(\Omega+\Lambda), \\
|w|=\operatorname{det}^{a}{ }_{\mu}=\operatorname{det}\left(e^{a}{ }_{\mu}+t^{a}{ }_{\mu}(\psi)\right), \quad t^{a}{ }_{\mu}(\psi)=\frac{\kappa^{2}}{2 i}\left(\bar{\psi} \gamma^{a} \partial_{\mu} \psi-\partial_{\mu} \bar{\psi} \gamma^{a} \psi\right),
\end{gathered}
$$

where $w^{a}{ }_{\mu}(x)$ invertible and $\Omega$ are the unified vierbein and the the unified scalar curvature of SGM spacetime, respectively. Accordingly $s_{\mu v} \equiv w^{a}{ }_{\mu} \eta_{a b} w^{b}{ }_{v}$ and $s^{\mu v}(x) \equiv w^{\mu}{ }_{a}(x) w^{v a}(x)$ are unified metric tensors of SGM spacetime[2,3]. The explicit expression of $\Omega$ is obtained by just replacing $e^{a}{ }_{\mu}(x)$ by $w^{a}{ }_{\mu}(x)$ in Ricci scalar $R$ of EGR [3]. $e^{a}{ }_{\mu}$ for $\mathrm{SO}(3,1)$ and $t^{a}{ }_{\mu}(\psi)$ for $\mathrm{SL}(2 \mathrm{C})$ are the ordinary vierbein of EGR and the mimic vierbein composed of the stress-energy-momentum tensor of superons $\psi(x)$, respectively. $G$ is the Newton gravitational constant, $\Lambda$ is a small cosmological constant. $\kappa$ of NLSUSY VA model [4] with the dimension $(\text { length })^{4}$ is now related to $\kappa^{2}=\left(\frac{c^{4} \Lambda}{8 \pi G}\right)^{-1}$. Remarkably $\kappa$, i.e. the strength of the superon(NG fermion)-vacuum coupling constant is determined by the cosmological quantities of spacetime $G$ and $\Lambda$ in the NLSUSY GR(SGM) scenario. SGM contains hierarchic two mass scales, $\frac{1}{G}$ (Planck scale) in the first term and $\kappa^{2} \sim \frac{G}{\Lambda}$ in the second term describing the curvature energy and the vacuum energy of SGM, respectively.

NLSUSY GR action (1.1) is invariant under the following new NLSUSY transformation[5];

$$
\delta_{\zeta} \psi=\frac{1}{\kappa} \zeta-i \kappa\left(\bar{\zeta} \gamma^{\rho} \psi\right) \partial_{\rho} \psi, \quad \delta_{\zeta} e^{a}{ }_{\mu}=i \kappa\left(\bar{\zeta} \gamma^{\rho} \psi\right) \partial_{[\rho} e^{a}{ }_{\mu]},
$$

consequently GL(4R) transformations on $w^{a}{ }_{\mu}$

$$
\delta_{\zeta} w^{a}{ }_{\mu}=\xi^{v} \partial_{\nu} w^{a}{ }_{\mu}+\partial_{\mu} \xi^{v} w^{a}{ }_{\nu}, \quad \delta_{\zeta} s_{\mu \nu}=\xi^{\kappa} \partial_{\kappa} s_{\mu \nu}+\partial_{\mu} \xi^{\kappa} s_{\kappa v}+\partial_{\nu} \xi^{\kappa} s_{\mu \kappa},
$$

where $\zeta$ is a constant spinor, $\partial_{[\rho} e^{a}{ }_{\mu]}=\partial_{\rho} e^{a}{ }_{\mu}-\partial_{\mu} e^{a}{ }_{\rho}$, and $\xi^{\rho}=i \kappa^{2}\left(\bar{\zeta} \gamma^{\rho} \psi\right)$. The commutators of two new NLSUSY transformations (1.3) on $\psi$ and $e^{a}{ }_{\mu}$ are GL(4R), i.e. new NLSUSY (1.3) is the square-root of GL(4R);

$$
\left[\delta_{\zeta_{1}}, \delta_{\zeta_{2}}\right] \psi=\Xi^{\mu} \partial_{\mu} \psi, \quad\left[\delta_{\zeta_{1}}, \delta_{\zeta_{2}}\right] e^{a}{ }_{\mu}=\Xi^{\rho} \partial_{\rho} e^{a}{ }_{\mu}+e^{a}{ }_{\rho} \partial_{\mu} \Xi^{\rho},
$$

where $\left.\Xi^{\mu}=2 i \kappa\left(\bar{\zeta}_{2} \gamma^{\mu} \zeta_{1}\right)-\xi_{1}^{\rho} \xi_{2}^{\sigma} e_{a}{ }^{\mu}\left(\partial_{[\rho} e^{a} \sigma\right]\right)$. They show the closure of the algebra. NLSUSY GR action (1.1) is invariant at least under the following spacetime symmetries[5] which is locally 
isomorophic to super-Poincaré(SP):

$[$ new NLSUSY $] \otimes[$ local GL $(4, R)] \otimes[$ local Lorentz $] \otimes[$ local spinor translation $($ LST $)]$

and the internal summetries for $\mathrm{N}$-superons $\psi_{j}(j=1,2, . . N)$ extended NLSUSY GR

$$
[\text { global SO}(\mathrm{N})] \otimes\left[\operatorname{local} \mathrm{U}(1)^{\mathrm{N}}\right]
$$

\section{Linearization of NL SUSY}

SGM action $L(w)$ (1.1) on SGM spacetime is unstable due to the global NLSUSY structure(SP d.o.f.) of the tangent spacetime at each curved spacetime point and breakes down spontaneously to EH Riemann spacetime action with superon(NG fermion) matter asfollows

$$
L(e . \psi)=-\frac{c^{4}}{16 \pi G}|e|\{R(e)+\Lambda+\tilde{T}(e, \psi)\}
$$

where $+\Lambda$ is the cosmological term necessary for reproducing NLSUSY VA action in the tangent spacetime and $\tilde{T}(e, \psi)$ is the kinetic and the gravitational interaction terms of superons. The second and the third terms produce N-etended NLSUSY VA action with $\kappa^{2}=\left(\frac{c^{4} \Lambda}{8 \pi G}\right)^{-1}$ in Riemannflat $\left(e^{a}{ }_{\mu}(x) \rightarrow \delta^{a}{ }_{\mu}\right)$ spacetime. We linearize SGM action (1.1) to obtain an equivalent and renormalizable field theory, where NLSUSY is recasted to the broken LSUSY defined on the LSUSY supermultiplet fields which are irreducible representations of SO(N) SP. As a preliminary for SGM linearization we have shown[6] that N=2 NLSUSY VA model (expanded in $\kappa$ ):

$$
L_{\mathrm{VA}}=-\frac{1}{2 \kappa^{2}}\left[1+\kappa^{2} t^{a}{ }_{a}+\frac{1}{2} \kappa^{4}\left(t^{a}{ }_{a} t^{b}{ }_{b}-t^{a}{ }_{b} t^{b}{ }_{a}\right)-\frac{1}{3 !} \kappa^{6} \varepsilon_{a b c d} \varepsilon^{e f g d} t^{a}{ }_{e} t^{b} t^{c}{ }_{g}+\cdots\right]
$$

invariant under N=2 NLSUSY transformation $\delta_{Q} \psi_{L}^{i}=\frac{1}{\kappa} \zeta_{L}^{i}-i \kappa\left(\bar{\zeta}_{L} \gamma^{a} \psi_{L}-\bar{\zeta}_{R} \gamma^{a} \psi_{R}\right) \partial_{a} \psi_{L}^{i}$, is equivalent to the following free action of the spontaneouly broken N=2 LSUSY:

$$
L_{\mathrm{lin}}=\partial_{a} \phi \partial^{a} \phi^{*}-\frac{1}{4} F_{a b}^{2}+i \bar{\lambda}_{R i} \not \partial \lambda_{R i}+\frac{1}{2}\left(D^{I}\right)^{2}-\frac{1}{\kappa} \xi^{I} D^{I}
$$

where $\psi_{L i},(i=1,2)$ is superon fields, $w^{a}{ }_{b}=\delta_{b}^{a}+\kappa^{2} t^{a}{ }_{b}, t^{a}{ }_{b}=-i \bar{\psi}_{L} \gamma^{a} \partial_{b} \psi_{L}+i \bar{\psi}_{R} \gamma^{a} \partial_{b} \psi_{R}, \psi_{R i}=$ $C \bar{\psi}_{L i}^{T} . \quad \xi^{I},(I=1,2,3)$ are arbitrary real parameters of the induced global $\mathrm{SU}(2)(\mathrm{SO}(3))$ rotation $\left(\xi^{I}\right)^{2}=1$. The last term is the Fayet-Iliopoulos $D$ term indicating spontaneous SUSY breaking with the vacuum expectation value $D^{I}=\xi^{I} / \kappa$. In these arguments all fields of LSUSY supermultiplet are composites of superons $\psi_{L}^{i}$, e.g. $\phi(\psi)=\frac{1}{\sqrt{2}} i \kappa \xi^{I} \bar{\psi}_{R} \sigma^{I} \psi_{L}-\sqrt{2} \kappa^{3} \xi^{I} \bar{\psi}_{L} \gamma^{a} \psi_{L} \bar{\psi}_{R} \sigma^{I} \partial_{a} \psi_{L} \cdots$, $\lambda_{L i}(\psi)=i \xi^{I}\left(\psi_{L} \sigma^{I}\right)_{i}+\kappa^{2} \xi^{I} \gamma^{a} \psi_{R i} \bar{\psi}_{R} \sigma^{I} \partial_{a} \psi_{L} \cdots, A_{a}(\psi)=-\frac{1}{2} \kappa \xi^{I}\left(\bar{\psi}_{L} \sigma^{I} \gamma_{a} \psi_{L}-\bar{\psi}_{R} \sigma^{I} \gamma_{a} \psi_{R}\right) \cdots$, etc and the familiar N=2 LSUSY transformations on the component fields of the supermultiplet are reproduced in terms of the NLSUSY transformations on $\psi_{L}^{i}$ contained[6]. When $\xi^{1}=\xi^{3}=0$, the supermultiplet is the ordinary vector supermultiplet with the vector $\mathrm{U}(1)$ gauge field as expected. The fact that the compact $\mathrm{SU}(2)$, though global, is generated in Riemann-flat spacetime of $\mathrm{N}=2$ SGM (2.2) shows that the vacuum of (1.1) has rich structures favourable to SGM scenario, where all fields except graviton are the superon-composite eigenstates of spacetime. 


\section{Cosmology of NLSUSY GR}

SGM spacetime is unstable and spontaneously breaks down to Riemann spacetime with superon matter, which is the birth of the universe by the quantum effect. The variation of (2.1) with respect to $e^{a}{ }_{\mu}$ gives the equation of motion for $e^{a}{ }_{\mu}$ recasted as follows

$$
R_{\mu v}(e)-\frac{1}{2} g_{\mu v} R(e)=\frac{8 \pi G}{c^{4}}\left\{\tilde{T}_{\mu v}(e, \psi)-g_{\mu v} \frac{c^{4} \Lambda}{8 \pi G}\right\}
$$

where $\tilde{T}_{\mu v}(e, \psi)$ is the stress-energy-momentum tensor of superon(NG fermion) matter including the gravitational interactions and $-\frac{c^{4} \Lambda}{8 \pi G}$ can be interpreted as the energy density of empty spacetime, which is the negative constant vacuum energy density i.e. the dark energy density $\rho_{D}$ of nature. (The sign is fixed uniquely in (1.1) to reproduce VA action in flat spacetime.) While, on tangent spacetime, the low energy theorem of the particle physics gives the creation amplitude of superon(massless NG fermion matter) from the vacuum as follows

$$
<\psi_{\alpha}^{L}\left|J_{\beta}^{M \mu}\right| 0>=i \sqrt{\frac{c^{4} \Lambda}{8 \pi G}}\left(\gamma^{\mu}\right)_{\alpha \beta} \delta^{L M}+\cdots,
$$

where $J^{M \mu}=i \sqrt{\frac{c^{4} \Lambda}{8 \pi G}} \gamma^{\mu} \psi^{M}+\cdots$ is the conserved supercurrent obtained by applying the Noether theorem to NLSUSY VA action[7] and $\sqrt{\frac{c^{4} \Lambda}{8 \pi G}}$ is the coupling constant $g_{\text {sup-vac }}$ of superon with the vacuum. Further we have seen in the preceding section that the right hand side of (3.1), which is essentially NLSUSY VA action, is equivalent to the broken LSUSY action (2.3) with the vacuum expectation value of the auxiliary field $\left\langle D>\sim \sqrt{\frac{c^{4} \Lambda}{8 \pi G}} \sim M_{S U S Y}{ }^{2}\right.$ (the square of the SUSY breaking mass scale) giving mass $M_{S U S Y}$ to a component field of the (massless) LSUSY supermultiplet. We find the interesting relations in SGM(NL SUSY) among the mysterious quantities of the cosmology and the low energy particle physics: $\rho_{D} \sim \frac{\Lambda}{G} \sim(\text { fundamental length of tangent space of } S G M)^{-4}$ $\sim<D>^{2}$ and $g_{\text {sup }-v a c} \sim \sqrt{\frac{\Lambda}{G}} \sim<D>$. Suppose that the neutrino $\lambda(x)$ (not superon $\psi(x)$ ) acquires mass of the order of the spontaneous SUSY breaking, i.e. $m_{v}{ }^{2} \sim \sqrt{\frac{\Lambda}{G}}$, SGM predicts the observed value $\rho_{D}{ }^{o b s} \sim\left(10^{-12} \mathrm{GeV}\right)^{4}$. NLSUSY GR gives in general $\Lambda \sim M_{S U S Y}{ }^{2}\left(\frac{M_{S U S Y}}{M_{\text {Planck }}}\right)^{2}$. The large mass scales in the broken LSUSY action appear through the liniarization of $\tilde{T}_{\mu v}(e, \psi)$ accompanied by the generation of the (broken) compact gauge symmetry, which contains the mass scale $\Lambda^{-1}$. NLSUSY GR(or SGM from the composite viewpoint) can be easily generalized to extra spacetime dimensions.

\section{References}

[1] K. Shima, European Phys. J. C7, 341(1999).

[2] K. Shima, Phys. Lett. B501, 237(2001).

[3] K. Shima, M. Tsuda, Class. and Quantum. Grav. 19, 1(2002).

[4] D.V. Volkov and V.P. Akulov, Phys. Lett. B46, 109(1973).

[5] K. Shima and M. Tsuda, Phys. Lett. B507, 260(2001).

[6] K. Shima, M. Tsuda and Y. Tanii, Phys. Lett. B546, 162(2002).

[7] K. Shima, Phys. Rev. D.15, 2165(1977). 